\title{
Preface to special issue: Expressiveness in Concurrency 2008
}

\author{
THOMAS HILDEBRANDT ${ }^{\dagger}$ and DANIELE GORLA \\ ${ }^{\dagger}$ IT University of Copenhagen (ITU), Rued Langgaards Vej 7, 2300 Copenhagen, Denmark \\ Email: hilde@itu.dk \\ `Dip. di Informatica, 'Sapienza' Università di Roma, Via Salaria 113, 00198 Roma, Italy \\ Email: gorla@di.uniroma1.it
}

Received 20 October 2009

This issue of Mathematical Structures in Computer Science contains three papers selected from the 15th International Workshop on Expressiveness in Concurrency (EXPRESS'08) held on 23 August 2008 in Toronto (Canada) as a satellite event of CONCUR'08.

The aim of the EXPRESS workshops is to bring together researchers interested in the relations between various formal systems, with particular emphasis on the field of concurrency. More specifically, they focus on the comparison between programming concepts (such as concurrent, functional, imperative, logic and object-oriented programming) and between mathematical models of computation (such as process algebras, Petri nets, event structures, modal logics and rewrite systems) on the basis of their relative expressive power. The EXPRESS workshops were originally held as meetings of the HCM project EXPRESS, which had the same focus and was active between January 1994 and December 1997. The first three workshops were project meetings and took place in 1994, 1995 and 1996. Since 1997, the workshop has been organised as an independent event with a call for papers and a significant attendance from outside the project. Since 1998, it has been held as a satellite event of the CONCUR conference.

This issue contains the full version of one paper submitted to the workshop and the contributions of the two invited speakers, Michele Bugliesi and Gianluigi Zavattaro. The papers have been extended and revised for journal publication, and have undergone a reviewing process in accordance with the standards of Mathematical Structures in Computer Science.

The first paper is an investigation into the expressive power of a core set of security and network abstractions that provide high-level primitives for specifying the honest principals in a network, while at the same time enabling an analysis of the networklevel adversarial attacks that may be mounted by an intruder. Some bisimulation-based equivalences are introduced, and compared with each other, to obtain a direct measure of the discriminating power of the intruder, and hence of the expressiveness of the corresponding intruder model.

The second paper is an exploration into the expressive power of languages that naturally model biochemical interactions relative to languages that naturally model basic chemical reactions only. Molecular association is the mechanism that distinguishes the former from the latter. The language with molecular association is Turing universal, while the language 
without it is not. Moreover, Turing universality derives from the interplay between the molecular primitives of association and dissociation: if either association or dissociation is removed, the language is no longer Turing complete.

The final paper deals with modal and mixed transition systems, which are specification formalisms that allow a mixing of over- and under-approximation. The paper discusses three fundamental decision problems for such specifications: whether a set of specifications has a common implementation; whether an individual specification has an implementation; and whether all implementations of a particular specification are implementations of another one. The worst-case computational complexity for the modal and mixed cases is investigated for each of these decision problems.

\section{Acknowledgements}

We would like to thank the authors for their efforts in producing the extended versions contained in this journal issue. We are very grateful to the anonymous reviewers for their extremely careful reading, which has led to a remarkable improvement in the submitted papers; their help has been crucial in producing this journal issue. We would also like to thank the EXPRESS'08 program committee for their evaluation of earlier versions of these papers, and Catuscia Palamidessi, who has supported this special issue from its inception. Last but not least, we would like to offer a special thanks to the Editor-inChief of Mathematical Structures in Computer Science for the opportunity to publish these papers in this special issue of the journal.

Thomas Hildebrandt

Daniele Gorla

EXPRESS'08 co-chairs 